\title{
Adaptive Control Allocation for Powered Descent Vehicles
}

\author{
Justin S. Green* \\ NASA Langley Research Center, Hampton, Virginia, 23681 \\ Robert E. Lindberg Jr. ${ }^{\dagger}$ \\ University of Virginia, Charlottesville, Virginia, 22904
}

\begin{abstract}
The following work details a study into real-time failure adaptive control allocation method for powered descent vehicle systems. The motivation for this work is to enable future human and robotic missions utilizing a powered descent system to tolerate engine failures in flight without the loss of crew or assets. This study is conducted using a six degree-of-freedom trajectory simulation of a PDV experiencing either a loss of thrust or an engine stuck full on failure scenario. Sequential least squares in the frequency domain is used on-board to process inertial measurement unit (IMU) data and generate an estimate of the PDV plant model, which is then fed to the guidance and control system. Data used by the sequential least squares method is generated from an in-flight maneuver. The work herein focuses on determining a maneuver that is least impactful to the PDV trajectory and enables a suitable plant model estimate. A $1.5 \mathrm{~s}$ long maneuver with an amplitude of $\mathbf{5 \%}$ throttle is determined to provide suitable data for the sequential least squares method to estimate a plant model. A PDV implementing this method can adapt to a single engine failure and continue to reach its touchdown conditions.
\end{abstract}

\section{Nomenclature}

$A \quad=$ orthogonal multi-sine function amplitude

$a_{\text {sensed }}=$ sensed acceleration

$B \quad=$ orthogonal multi-sine function global amplitude

$\boldsymbol{B}=$ bias array

$C \quad=$ non-dimensionalized force or moment coefficient

$D_{\text {noz }} \quad=$ engine nozzle diameter

$F \quad=$ force

$\boldsymbol{F}_{\boldsymbol{c}} \quad=$ control command array

$f \quad=$ orthogonal multi-sine function targeted frequency

$G \quad=$ number of targeted frequencies used in the orthogonal multi-sine function

$h \quad=$ size of data operated on by the sequential least squares method

$\left[I_{x x}, I_{y y}, I_{z z}\right]=$ principle moments of inertia

$i \quad=$ measurement index

$J \quad=$ ordinary least squares cost function

$k=$ total number of measurements taken

$M \quad=$ moment

$m \quad=$ powered descent vehicle mass

$N=$ number of excitation functions used in the orthogonal multi-sine function

$[p, q, r] \quad=$ components of the powered descent vehicle rotation vector in the body coordinate system

$\boldsymbol{Q} \quad=$ powered descent vehicle plant model

$\boldsymbol{T} \quad=$ throttle array

*Aerospace Engineer, Atmospheric Flight and Entry Systems Branch, NASA Langley Research Center, Hampton, VA, 81. Doctoral Candidate, Dept. of Mechanical and Aerospace Engineering, University of Virginia, Charlottesville, VA, 22903.

$\dagger$ Visiting Research Professor, Dept. of Mechanical and Aerospace Engineering, University of Virginia, PO Box 400746, Charlottesville, VA, 22904, AAS Fellow, AIAA Fellow. 


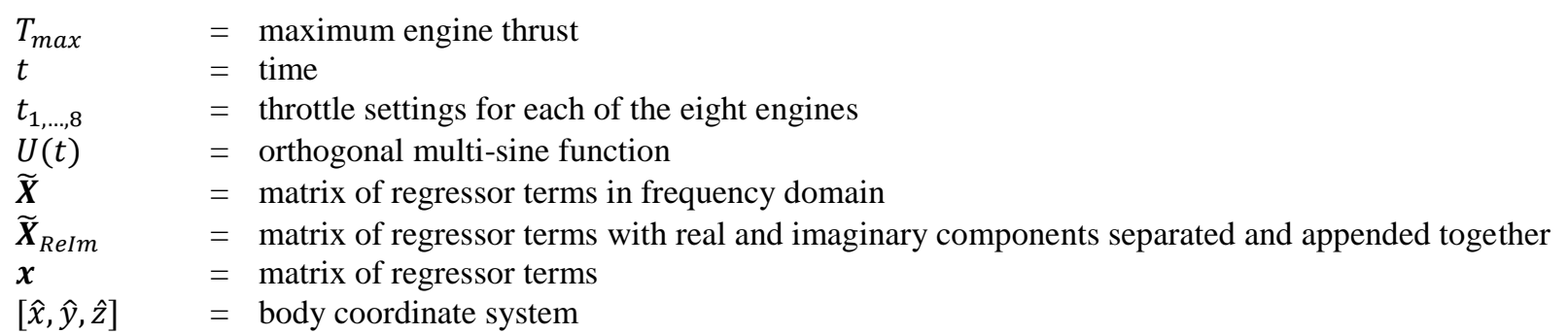

$\left[x_{I M U 2 C O M}, y_{I M U 2 C O M}, z_{I M U 2 C O M}\right]=$ inertial measurement to center of mass distances in the body coordinate system $\left[y_{1, \ldots, 8}, z_{1, \ldots, 8}\right]=x$ and $y$ body axis location for each of the eight engines

$Z \quad=$ measured data array

$\tilde{Z} \quad=$ measured data array in frequency domain

$\tilde{z}_{\text {ReIm }} \quad=$ appended measured data array with real and imaginary components separated and appended together

$\tilde{\boldsymbol{Z}} \quad=$ appended measured data array in frequency domain

$\begin{array}{ll}\theta & =\text { array of parameters } \\ \hat{\theta} & =\text { parameter estimate } \\ \tilde{v} & =\text { equation errors in the frequency domain } \\ \tilde{\boldsymbol{v}} & =\text { appended equation errors in the frequency domain } \\ \sigma^{2} & =\text { variance } \\ \phi & =\text { orthogonal multi-sine function phase shift } \\ \omega & \end{array}$

\section{Subscripts}

all $=$ all parameter estimates

$b \quad=$ bias term

c $\quad=$ commanded

$C_{m y} \quad=$ values pertaining to the pitching moment coefficient

$C_{m z} \quad=$ values pertaining to the yawing moment coefficient

$i, k=$ indices

$m \quad$ = moment

$[x, y, z]=$ body coordinate directions

\section{Introduction}

$\mathrm{T}$

he goal of this research is to enable a powered descent vehicle (PDV) to adapt in real-time to failures and degradations in its performance that change its dynamic behavior. Past robotic Mars missions have not had this capability. For example, during the Mars Science Laboratory (MSL) mission, the descent stage designers performed extensive testing on the Mars Lander Engines (MLEs) and Reaction Control System (RCS) thrusters and found that failures in these systems were unlikely ${ }^{1}$. This along with mission constraints led the designers to conclude that the MSL "spacecraft was not designed to survive an engine failure for either the MLEs or RCS thrusters". The risk inherent in this approach is acceptable for a mission and descent system with a single payload or asset. However, this approach is unacceptable when a mission and descent system must consider the safety and security of already established assets on the ground and/or onboard crew members. Therefore, redundancy and failure mitigation are of concern for PDVs. Implementing redundancies for larger systems, such as descent engines, can be problematic due to the mass or the complexity of adding such systems. Constraints from the mission itself can also hinder the implementation of redundant engines and supporting systems.

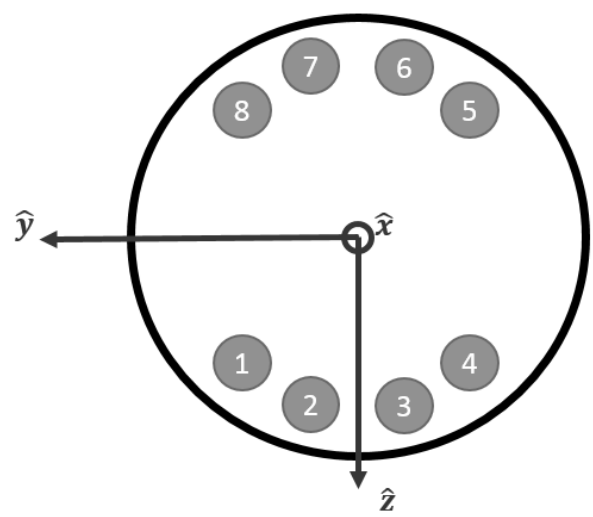

Figure 1. Orientation of the eight thrusters with respect to the vehicle body frame. 
Human Mars mission architectures, due to their early stages in design and development, are also in need of component failure mitigation strategies that do not add significant mass and complexity. One solution for mitigating these issues would be to build a guidance and control strategy that can adapt and reconfigure in the face of component failures. Such a capability has been identified as a key technology to be developed by NASA Space Technology Roadmap for Entry, Descent, and Landing (EDL) ${ }^{3}$. A guidance and control strategy of this nature would enable a descent system to land in the event of a component failure without the loss of crew or assets.

This paper provides an overview of a real-time strategy for updating a PDV plant model on-board. This strategy aides the guidance and control system's ability to maximize its control authority in the event of an engine failure. Section II describes the simulation environment, and the PDV used to demonstrate the failure mitigation strategy. Section III discusses the use of real-time parameter identification, which is the basis of the work herein. Section IV details the derivation of the plant model form and how the on-board estimates are generated. Section V contains the investigations performed and the results obtained. Section VI expands the failure mitigation strategy to other failure scenarios, and Section VII provides the final conclusions.

The six degree-of-freedom (DoF) simulation is built using the Program to Optimize Simulated Trajectories - II (POST2) software ${ }^{4}$. In this simulation, the PDV model is based on a human Mars mission architecture outlined by NASA's evolvable Mars campaign ${ }^{5,6}$. The PDV initial mass is $46.4 \mathrm{mt}$ (of which 9.5 $\mathrm{mt}$ is fuel) and utilizes eight fixed engines with a maximum thrust of $100 \mathrm{kN}$ per engine for deceleration ${ }^{5,6}$. The eight engines, shown in Figure 1, are independently throttled to create a differential thrust that pitches and yaws the PDV, and enables it to follow a gravity turn trajectory supplied by an onboard guidance routine. The PDV simulation begins at a planetodetic altitude of $3559 \mathrm{~m}$, planet relative velocity of 471 $\mathrm{m} / \mathrm{s}$, and a flight path angle of $19.9^{\circ}$. The trajectory targets the beginning of the vertical descent phase for landing, which is $2.5 \mathrm{~m} / \mathrm{s}$ at $12.5 \mathrm{~m}$ above ground. Through the vertical descent phase, the PDV reaches touchdown, $0 \mathrm{~m}$, at 2.5 $\mathrm{m} / \mathrm{s}$. The simulation assumes no winds or aerodynamics are acting on the PDV. Additionally, Mars Global Reference Atmospheric Model (Mars-GRAM)

2010

\section{Trajectory Simulation}
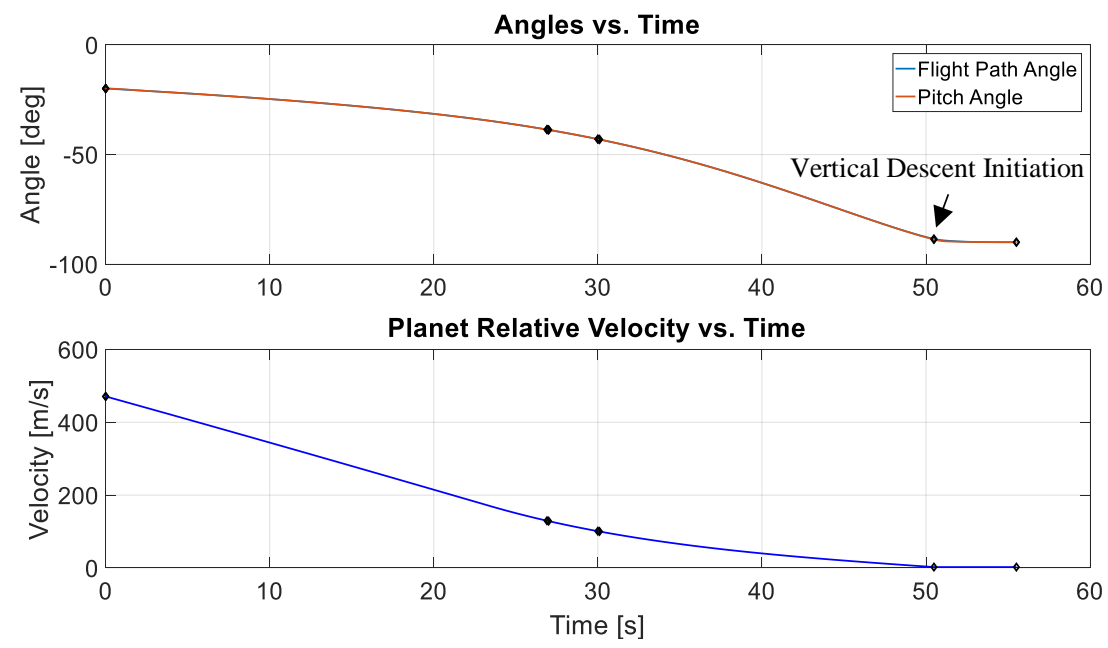

Figure 2. Thrust and throttle profiles of the nominal PDV trajectory.
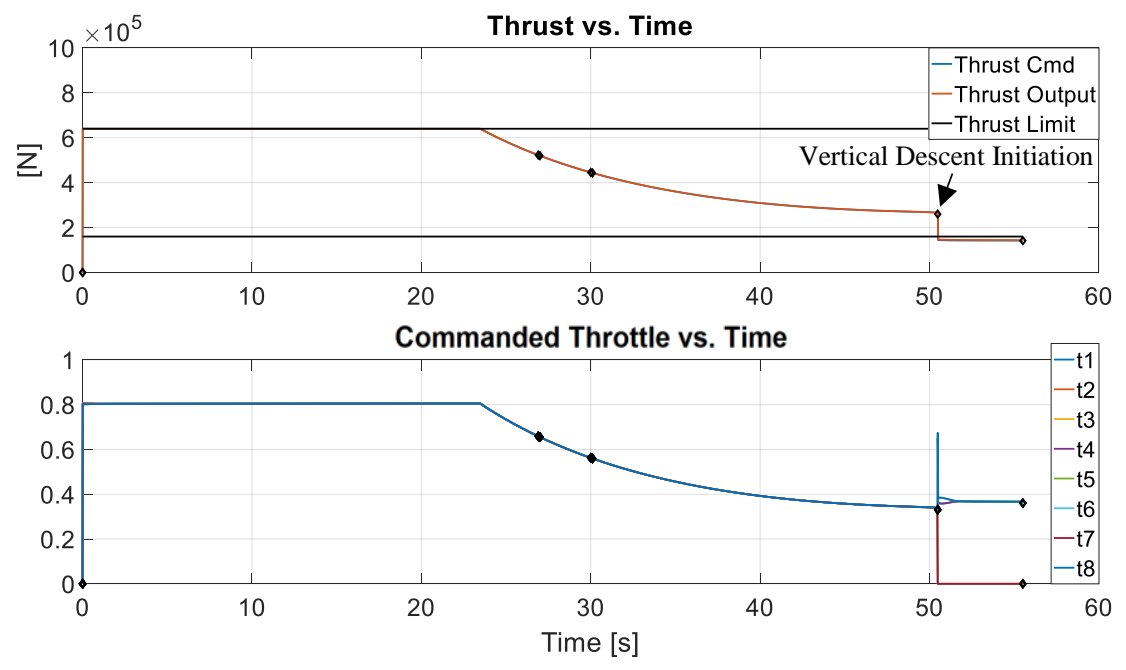

Figure 3. Nominal trajectory of the PDV. The gravity turn phase operates between 0 and $51 \mathrm{~s}$; the vertical descent phase operates from $51 \mathrm{~s}$ until touchdown. 
atmosphere and $\mathbf{J} 2$ gravity harmonic are used ${ }^{7}$.

Inertial measurement unit (IMU) and IMU propagator models are used to simulate the sensor and navigation systems on the PDV. These models were developed and used for the Low Density Supersonic Decelerator (LDSD) project $^{8}$.

The PDV trajectory is divided into two phases: gravity turn and vertical descent. The gravity turn phase assumes the PDV thrust vector is aligned with the PDV velocity vector. This assumption is enforced by pitching the PDV as needed through differential throttling of the engines. During this phase, differential throttling is also used to keep the vehicle travelling in plane relative to its initial velocity azimuth. The enforcement of these assumptions are shown in Figure 2 and Figure 3. Note in Figure 3, that the guidance commanded thrust stays within 20-80\% of the maximum engine thrust of $100 \mathrm{kN}$ per engine. This leaves room for the control system to operate. Switching to the vertical descent phase maintains the PDV constant rate of descent while removing lateral motion. At the beginning of vertical descent, four of the engines are shutdown (engines 2, 3,6 and 7). This is done to keep throttle commands from dropping below a $20 \%$ threshold.

\section{Real-Time Parameter Identification}

System and parameter identification is the determination of model form and value based on imperfect observations of the inputs and outputs of a desired system for the purpose of generating an equivalent mathematical surrogate. An equivalent mathematical surrogate is the simplest model that exhibit the desired system characteristics ${ }^{9}$. In the past, system and parameter identification has been used to characterize and develop models for aircraft, turbines, and rocket engines $^{9,10}$. For these applications, data was collected during a test and then processed off-line. Real-time approaches have been developed for fault detection and enabling fault tolerant control in aircraft ${ }^{11,12}$.

Real-time parameter identification, specifically sequential least squares in the frequency domain (SLSFD), is used here to fulfill the research goal of an adaptable PDV control system. This strategy is implemented on-board the PDV in the six DoF trajectory simulation. It allows the PDV to update its internal plant model and identifies failed or underperforming engines. The parameter identification equations described herein, Eqs. (1-9), are taken from Klein and Morelli, Ref. 8.

\section{A. Ordinary Least Squares}

Real-time parameter identification methods provide on the fly analysis of a systems behavior, which are used to estimate the parameter values inside an existing model. The recursive ordinary least squares cost function, Eq. (1), forms the basis of the approach taken in the work herein.

$$
J(\theta)=\frac{1}{2} \sum_{i=1}^{k}\left[z(i)-\boldsymbol{x}^{T}(i) \theta\right]^{2}
$$

Where $z$ is the measured data array that the model will be based on, $\boldsymbol{x}$ is a matrix of regressor terms, $\theta$ is the array of parameters to be estimated, and $i$ is the index of measurements, and $k$ is the total number of measurements taken. The parameters can be solved for by minimizing this cost function, which leads to

$$
\hat{\theta}=\left(\boldsymbol{x}^{T} \boldsymbol{x}\right)^{-1} \boldsymbol{x}^{T} Z
$$

where $\hat{\theta}$ is an array of parameter estimates.

\section{B. Sequential Least Squares in the Frequency Domain}

The implementation, used here, of SLSFD utilizes the Euler approximation of the discrete Fourier transform of both the regressor matrix and the measured data

$$
\begin{gathered}
\widetilde{\boldsymbol{X}}_{i}(\omega)=\widetilde{\boldsymbol{X}}_{i-1}(\omega)+\boldsymbol{x}_{i}(i) e^{-j \omega i \Delta t} \\
\tilde{z}_{i}(\omega)=\tilde{z}_{i-1}(\omega)+z_{i}(i) e^{-j \omega i \Delta t} \quad i=1, \ldots, h
\end{gathered}
$$

where $j=\sqrt{-1}$ and $\omega$ is the angular frequency. The rigid body dynamics that are of interest occupy a frequency band $<12 \mathrm{~Hz}$. With a limited frequency band of interest, Eqs. (3-4) efficiently compute the discrete Fourier transform. Moreover, keeping the investigation within this limited band, allows for automatic filtering of wideband measurement noise. Through Eqs. (3-4), the ordinary least squares cost function then changes to 


$$
J(\theta)=\frac{1}{2}(\tilde{z}-\widetilde{\boldsymbol{X}} \theta)^{\dagger}(\tilde{z}-\widetilde{\boldsymbol{X}} \theta)
$$

where $\dagger$ signifies the transpose of the complex conjugate. Solving for the minimum of the cost function yields

$$
\begin{gathered}
\hat{\theta}=\left(\widetilde{\boldsymbol{X}}_{\text {ReIm }}{ }^{\dagger} \widetilde{\boldsymbol{X}}_{\text {ReIm }}\right)^{-1}\left(\widetilde{\boldsymbol{X}}_{\text {ReIm }}{ }^{\dagger} \tilde{Z}_{\text {ReIm }}\right) \\
\operatorname{Cov}(\hat{\theta})=\sigma^{2}\left(\widetilde{\boldsymbol{X}}_{\text {ReIm }}{ }^{\dagger} \widetilde{\boldsymbol{X}}_{\text {ReIm }}\right)^{-1}
\end{gathered}
$$

where

$$
\begin{aligned}
\widetilde{\boldsymbol{X}}_{\text {ReIm }} & =\left[\begin{array}{l}
\operatorname{Re}(\widetilde{\boldsymbol{X}}) \\
\operatorname{Im}(\widetilde{\boldsymbol{X}})
\end{array}\right] \\
\tilde{Z}_{\text {ReIm }} & =\left[\begin{array}{l}
\operatorname{Re}(\tilde{z}) \\
\operatorname{Im}(\tilde{z})
\end{array}\right]
\end{aligned}
$$

Appending the real and imaginary parts of the regressor and measurement arrays, as seen in Eqs. (8-9), effectively doubles the data content available to estimate the parameter array, $\hat{\theta}$. The sequential least squares method operates on sections of data of size $h$, which depends on the time window of interest. The covariance provides a measure of the relationship between different terms in $\widetilde{\boldsymbol{X}}$. If the off diagonal terms are larger in magnitude than the diagonal terms, then the data is correlated. This can lead to issues in estimating the parameters in $\hat{\theta}$, and will require efforts to decorrelate the terms in the covariance matrix.

\section{Plant Model Generation of the Powered Descent Vehicle}

\section{A. Thrust Selection}

The PDV follows total force, pitching and yawing moment commands that are computed from the guidance and control routines. These commands are met through differential thrusting of the eight fixed engines. Note that rolling moment and normal forces are not controllable via differential throttling. Fortunately, the disturbances in these dimensions should be small and are assumed controlled by other means. Given the positions of the engines, a formulation for how they meet these commands is

$$
\begin{aligned}
& F_{c}=T Q+B \\
& \boldsymbol{F}_{c}=\left[F_{x c} M_{y c} M_{z c}\right] \\
& \boldsymbol{T}=\left[t_{1} t_{2} t_{3} t_{4} t_{5} t_{6} t_{7} t_{8}\right] \\
& \boldsymbol{Q}=T_{\max }\left[\begin{array}{lll}
\hat{\theta}_{1} & z_{1} \hat{\theta}_{1} & y_{1} \hat{\theta}_{1} \\
\hat{\theta}_{2} & z_{2} \hat{\theta}_{2} & y_{2} \hat{\theta}_{2} \\
\hat{\theta}_{3} & z_{3} \hat{\theta}_{3} & y_{3} \hat{\theta}_{3} \\
\hat{\theta}_{4} & z_{4} \hat{\theta}_{4} & y_{4} \hat{\theta}_{4} \\
\hat{\theta}_{5} & z_{5} \hat{\theta}_{5} & y_{5} \hat{\theta}_{5} \\
\hat{\theta}_{6} & z_{6} \hat{\theta}_{6} & y_{6} \hat{\theta}_{6} \\
\hat{\theta}_{7} & z_{7} \hat{\theta}_{7} & y_{7} \hat{\theta}_{7} \\
\hat{\theta}_{8} & z_{8} \hat{\theta}_{8} & y_{8} \hat{\theta}_{8}
\end{array}\right] \\
& \boldsymbol{B}=T_{\max }\left[\hat{\theta}_{b x} \hat{\theta}_{b y} \hat{\theta}_{b z}\right]
\end{aligned}
$$

where $F_{x c}, M_{y c}$, and $M_{z c}$ are the individual commands. Throttle solutions for each engine $t_{1-8}$ meet the command array, $\boldsymbol{F}_{\boldsymbol{c}}$. The plant model, $\boldsymbol{Q}$, is comprised of the maximum thrust, $T_{\max }$; the estimated engine efficiency (or parameter), $\hat{\theta}_{1-8}$, for each engine; and the moment arm of each engine, $y_{1-8}$ and $z_{1-8}$. The bias array, $\boldsymbol{B}$, captures unmodeled dynamics, by including the bias parameter estimates, which correspond to the force, pitching moment, and yawing moment, respectively. To solve for the throttle array, $\boldsymbol{T}$, Eq. (10a) can be reformulated into an ordinary least squares problem 


$$
\boldsymbol{T}=\left[\boldsymbol{F}_{\boldsymbol{c}}-\boldsymbol{B}\right] \boldsymbol{Q}^{T}\left[\boldsymbol{Q} \boldsymbol{Q}^{T}\right]^{-1}
$$

\section{B. Plant Model Generation}

The parameter estimates used in Eq. (10d), are determined through the SLSFD method described above. Initially, separate models for the total force, $F_{x}$; pitching moment, $M_{y}$; and yawing moment, $M_{z}$, are created. The nondimensionalized versions of these models are

$$
\begin{gathered}
C_{x}=\frac{F_{x}}{T_{\text {max }}}=\sum_{i=1}^{8} \theta_{i} t_{i}+\theta_{b x} \\
C_{m y}=\frac{M_{y}}{T_{\text {max }} D_{\text {noz }}}=\sum_{i=1}^{8} \theta_{i} \frac{z_{i} t_{i}}{D_{\text {noz }}}+\theta_{b y} \\
C_{m z}=\frac{M_{z}}{T_{\text {max }} D_{\text {noz }}}=\sum_{i=1}^{8} \theta_{i} \frac{y_{i} t_{i}}{D_{\text {noz }}}+\theta_{b z}
\end{gathered}
$$

where $D_{n o z}$ is the engine nozzle diameter and is included for non-dimensionalization purposes.

Values of $F_{x}, M_{y}$, and $M_{z}$ are estimated through an understanding of the PDV equations of motion and IMU data.

$$
\begin{gathered}
F_{x}=m\left(a_{\text {sensed }_{x}}-\left(q^{2}+r^{2}\right) x_{I M U 2 C o M}+(p q-\dot{r}) y_{I M U 2 C o M}+(p r+\dot{q}) z_{I M U 2 C o M}\right) \\
M_{y}=\dot{q} I_{y y}+p r\left(I_{x x}-I_{z z}\right)+m z_{I M U 2 C o M} a_{\text {sensed }_{x}}-m x_{I M U 2 C o M} a_{\text {sesned }_{z}} \\
M_{z}=\dot{r} I_{z z}+p q\left(I_{y y}-I_{x x}\right)+m x_{I M U 2 C o M} a_{\text {sensed }_{y}}-m y_{I M U 2 C o M} a_{\text {sesned }_{x}}
\end{gathered}
$$

The PDV principal moments of inertia (MoI) are given by $I_{x x}, I_{y y}$, and $I_{z z}$. The cross products of inertia are assumed to be negligibly small and are ignored. The distance between the IMU position and the center of mass (CoM) in the body frame is defined by $x_{\text {IMU2CоM }}, y_{\text {IMU2CoM }}$, and $z_{\text {IMU2CоM }}$. The IMU provides the PDV attitude rates and accelerations about the roll, $p$; pitch, $q$; and yaw, $r$, axes. Additionally, the IMU provides the translational acceleration data $a_{\text {sense }_{x}}, a_{\text {sensed }_{y}}$, and $a_{\text {sesned }_{z}}$. Principal

With values of $F_{x}, M_{y}$, and $M_{z}$ determined, Eqs. (12-14) can be transformed into the frequency domain and set up as SLSFD problems.

$$
\begin{gathered}
\tilde{z}_{C_{x}}=\hat{\theta} \widetilde{\boldsymbol{X}}_{C_{x}}+\widetilde{v}_{C_{x}} \\
\tilde{z}_{C_{m y}}=\hat{\theta} \widetilde{\boldsymbol{X}}_{C_{m y}}+\widetilde{v}_{C_{m y}} \\
\tilde{z}_{C_{m z}}=\hat{\theta} \widetilde{\boldsymbol{X}}_{C_{m z}}+\tilde{v}_{C_{m z}}
\end{gathered}
$$

The arrays $\tilde{z}_{C_{x}}, \tilde{z}_{C_{m y}}$, and $\tilde{z}_{C_{m z}}$ are the frequency content of the non-dimensionalized force and moments in Eqs. (1214). The two dimensional arrays $\widetilde{\boldsymbol{X}}_{C_{x}}, \widetilde{\boldsymbol{X}}_{C_{m y}}$, and $\widetilde{\boldsymbol{X}}_{C_{m z}}$ are the frequency content of the regressor terms in Eqs. (1214). The arrays $\tilde{v}_{C_{x}}, \tilde{v}_{C_{m y}}$, and $\tilde{v}_{C_{m z}}$ are the complex equation errors in the frequency domain. The arrays in Eqs. (1820) can be appended to one another to create

$$
\begin{gathered}
\tilde{\boldsymbol{z}}=\hat{\theta} \widetilde{\boldsymbol{X}}+\tilde{\boldsymbol{v}} \\
\tilde{\boldsymbol{z}}=\left[\begin{array}{c}
\tilde{z}_{C_{x}} \\
\tilde{z}_{C_{m y}} \\
\tilde{z}_{C_{m z}}
\end{array}\right] \\
\widetilde{\boldsymbol{X}}=\left[\begin{array}{c}
\widetilde{\boldsymbol{X}}_{C_{x}} \\
\widetilde{\boldsymbol{X}}_{C_{m y}} \\
\widetilde{\boldsymbol{X}}_{C_{m z}}
\end{array}\right]
\end{gathered}
$$




$$
\tilde{\boldsymbol{v}}=\left[\begin{array}{c}
\tilde{v}_{C_{x}} \\
\tilde{v}_{C_{m y}} \\
\tilde{v}_{C_{m z}}
\end{array}\right]
$$

The creation of Eq. (21) combines all the available data content into a single estimate, which allows for more accurate estimation of the values in $\hat{\theta}$ than using Eqs. (18-20) individually. The parameter estimates in $\hat{\theta}$ can be found using Eq. (6). Note, that the conversion into the frequency domain removes the bias. So after solving Eq. (6), $\hat{\theta}_{b x}, \hat{\theta}_{b y}$, and $\hat{\theta}_{b z}$ are found separately by solving a second ordinary least squares problem in the time domain, Eq. (2), for each force and moment. The formulation of the plant model is made such that a PDV operating under nominal conditions (i.e. no engine failures) would have parameter estimates, $\hat{\theta}$, of all ones, and the three bias parameters all equal to zero. A PDV experiencing a loss of thrust in a single engine would have a $\hat{\theta}$ array of all ones except for the engine that has failed, which would have a value of zero.

\section{Orthogonal Multi-Sines Input}

Early investigations in applying the above analysis quickly identified high correlation between the eight engine throttle commands. These correlations resulted in poor parameter estimates that were unusable for meeting the goal of the present research. The solution to this issue is to inject a test input on top of the throttle command solutions, which is referred to as a maneuver for the rest of this paper. Orthogonal multi-sine functions generate functions that are orthogonal in time and frequency, which decorrelates the throttle commands, thus allowing the effects of each engine to be uniquely identified. Orthogonal multi-sine waveforms were generated for each of the eight engines using the MKMSSWP function within the System IDentification Programs for AirCraft (SIDPAC) ${ }^{13}$. This function generates waveforms that are a sum of sinusoids at discrete frequencies that are phase optimized to minimize deviations from the nominal input, known as the peak factor. The form of these sinusoids are

$$
U_{i}(t)=B_{i} \sum_{k=1}^{G_{i}} A_{i, k} \sin \left(2 \pi f_{i, k} t+\phi_{i, k}\right) ; i=1,2, \ldots, N
$$

where $N$ is the number of excitation functions to be generated, and $G_{i}$ is the number of targeted frequencies, $f_{i, k}$, in each function. Through optimization, each excitation function targets a minimum peak factor by adjusting the function amplitude, $A_{i, k}$, and the phase shift, $\phi_{i, k}$, for each target frequency. A global amplitude, $B_{i}$, is applied to each excitation function, $U_{i}$, to meet the needs of the maneuver. A detailed description of the generation and use of orthogonal multisine functions can be found in Ref. 14.

\section{Analysis}

The goal of this research is to enable a PDV to adapt in real-time to failures and degradations in its performance. The failure scenarios studied in this report are single engine failures where the engine either loses all thrust, or is stuck full on. The objective of the real-time parameter identification, is to identify the type of failure, approximate the magnitude of the engine performance degradation, and feed that information to the guidance and control system in the form of a plant model update. This update enables the PDV to change its throttle commands according to the degradation in the performance, and allow the PDV to continue its flight and reach its target conditions.

The inclusion of a maneuver requires an understanding of how the throttle amplitude and duration affect the parameter estimates and the PDV's ability to reach its target conditions in the event of a failure. Additionally, the effects of IMU errors on these same metrics need to be well understood.

\section{A. Maneuver Study}

The study into the maneuver configuration investigates the throttle amplitude and maneuver time length. The goal for this study is to determine the least disruptive maneuver combination that provides the best

\section{Table 1. Maneuver design space.}

\begin{tabular}{l|l} 
Maneuver Throttle Multiplier, \%/100 & {$[0.3,0.2,0.1,0.05]$} \\
\hline Maneuver Length of Time, s & {$[4.0,3.5,3.0,2.5,2.0,1.7,1.5]$}
\end{tabular}

possible outcome for the PDV (i.e. provides a reasonable PDV plant model that enables the PDV to reach its target conditions in the event of an engine failure). For each maneuver time length a unique group of eight orthogonal multisine waveforms were generated using the SIDPAC toolbox. When finding the minimum peak factor, the optimization used within SIDPAC resulted in local minima solutions. Thus 10 versions of each maneuver length of time, shown in 
Table 1, were created. This enabled a study into the effects of the maneuver time length itself, and not just the result of a particular local minima solution. Table 1 provides the design space exploration of the maneuvers. Permutations of these maneuver combinations are combined with each engine failure scenario for all eight engines.

The focus of the maneuver study is on the effects they have on the parameter estimates and the PDV's ability to target landing conditions; not the logic for triggering the maneuver itself. Therefore, for this study, the initiation of the maneuver is assumed to be concurrent with the beginning of the failure. This is equivalent to instantanous detection of a failure, without knowledge of what type of failure has occurred.

As an example, Figure 4 shows box-and-whiskers plots of the plant model fit error versus the maneuver multiplier and time length for the failure scenario where engine four has lost all thrust. Similar analyses with similar results were performed for each of the engines. Plant model fit error is the root mean square error and is defined as

$$
\begin{gathered}
\text { Fit Err. }=\sqrt{\frac{\sum_{k=1}^{11}\left(\hat{\theta}_{\text {all }}(i)-\theta_{\text {all }}(i)\right)^{2}}{11}} \\
\hat{\theta}_{\text {all }}=\left[\begin{array}{llllllllllll}
\hat{\theta}_{1} & \hat{\theta}_{2} & \hat{\theta}_{3} & \hat{\theta}_{4} & \hat{\theta}_{5} & \hat{\theta}_{6} & \hat{\theta}_{7} & \hat{\theta}_{8} & \hat{\theta}_{b x} & \hat{\theta}_{b y} & \hat{\theta}_{b z}
\end{array}\right] \\
\theta_{\text {all }}=\left[\begin{array}{lllllllllll}
\theta_{1} & \theta_{2} & \theta_{3} & \theta_{4} & \theta_{5} & \theta_{6} & \theta_{7} & \theta_{8} & \theta_{b x} & \theta_{b y} & \theta_{b z}
\end{array}\right]
\end{gathered}
$$

The box-and-whisker provides a rough statistical representation of the 10 versions of each maneuver time length. In general, this figure shows the trend that increasing the maneuver multiplier and time length lead to decreased model fit error. This trend is observed, because least squares methods depend on the data content. Larger maneuver amplitudes increase the amount of data that is above the noise threshold, and longer maneuvers add more data for the least squares method to operate on. Therefore, increasing the maneuver multiplier and time length increase the data content for the SLSFD method to use, thus lowering the model fit error.

Figure 5 is a sample of the results from the maneuver study. For brevity, only one engine failure and scenario (engine four full loss of thrust) along with two of the throttle multipliers (0.05 and 0.3$)$ is shown. Figure 5 is organized in increasing maneuver throttle multiplier and show the PDV targeting conditions. These figures show the PDV conditions planet relative velocity, flight path angle, and pitch angle at the initiation of vertical descent (left column) and at touchdown (right column). The $x$ axis of each plot correspond to the different maneuver lengths of time studied. Within each figure the black dashed line represents the nominal flight of the PDV, where no failure occurred. The green diamonds represent a PDV experiencing a failure, without implementing a maneuver and updating its plant model. The box-and-whisker plots (in blue and red) show the result of a PDV implementing the corresponding maneuver time length and multiplier. The box-and-whisker plots provide a rough statistical interpretation of the 10 versions of the maneuver time length.

In looking at the results in Figure 5, it can be seen that implementing the real-time parameter identification using any of the maneuvers significantly improved the PDV's ability to reach the target conditions. In Figure 5a, there is a noticeable step increase in performance between the 2 and $2.5 \mathrm{~s}$ maneuvers. This step is attributed to the step improvement in the fit error shown in Figure 4. However, it should be noted that the step improvement seen at vertical descent initiation (left column in Figure 5a) does not translate into significant differences in meeting the touchdown
Eng. 4 Zero Thrust

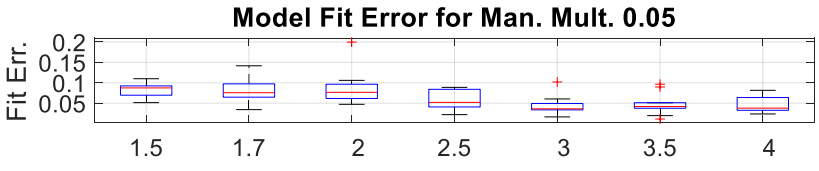

Man. Mult. 0.1

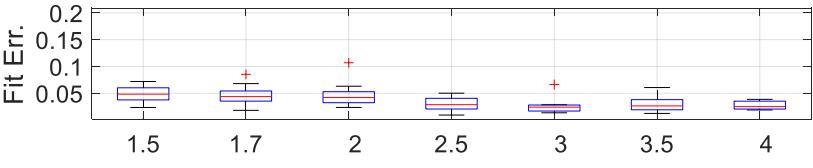

Man. Mult. 0.2
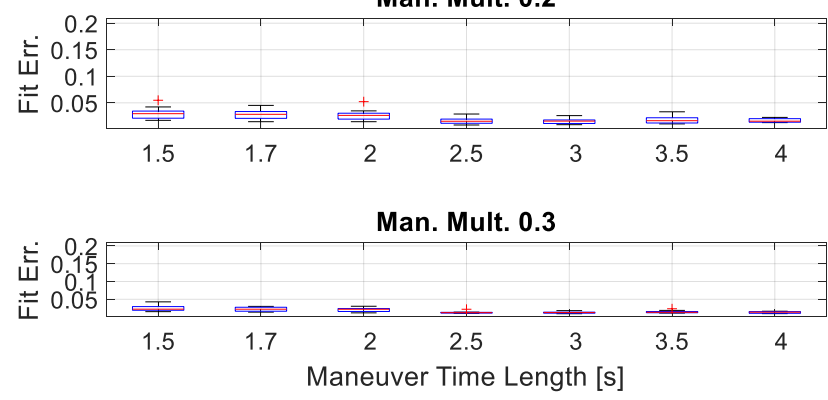

Figure 4. Model fit error versus maneuver multiplier and maneuver time length. Models are generated for a PDV experience a complete loss of thrust in engine four. The box-and-whiskers plots divided by: Top Whisker (95\%-tile), Top of Box (75\%-tile), Red Line (50\%-tile), Bottom of Box (25\%-tile), and Bottom Whisker (5\%tile). Outliers are indicated by a red cross. 
Eng. 4 Zero Thrust
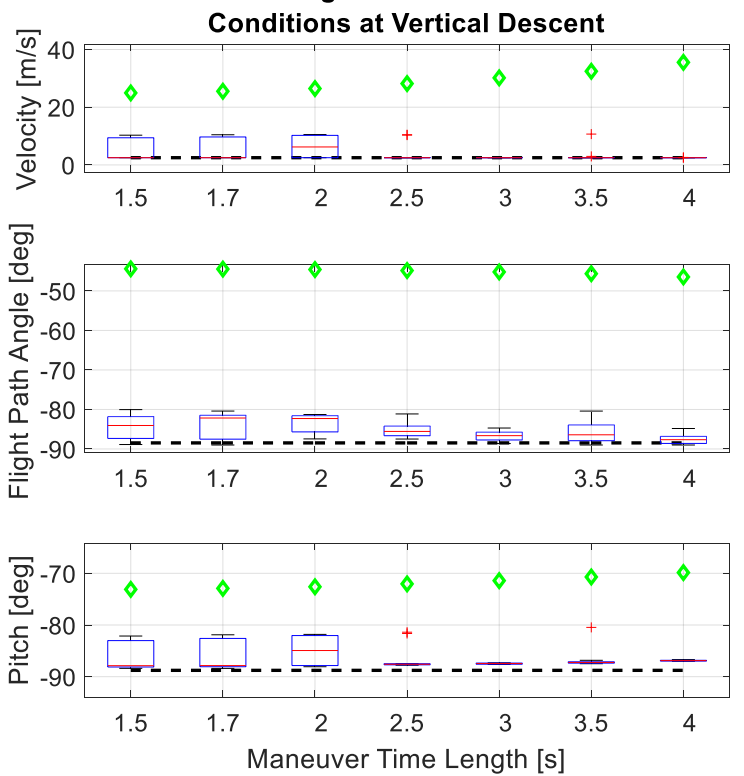

a)
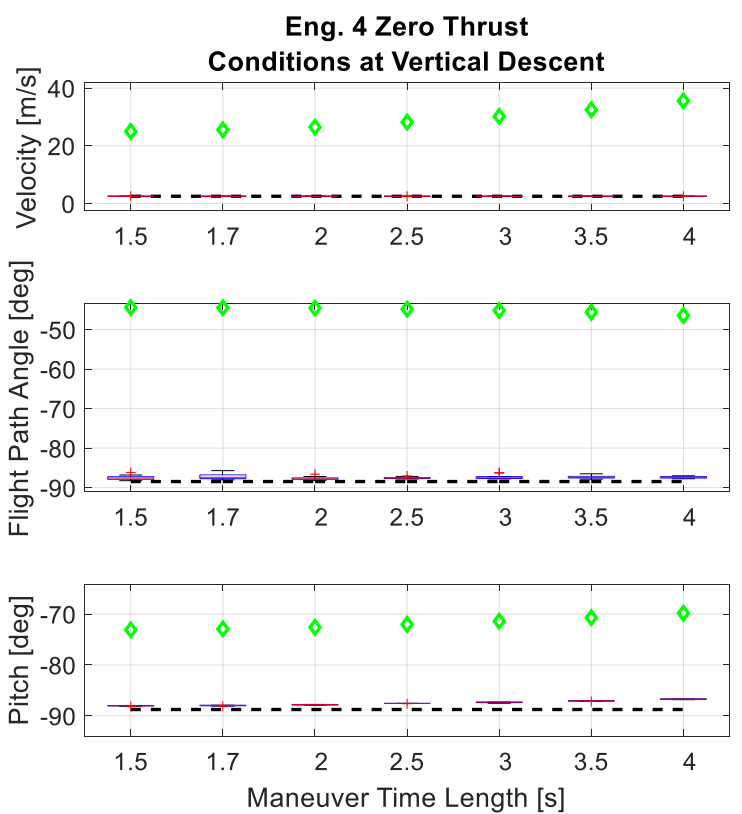

b)
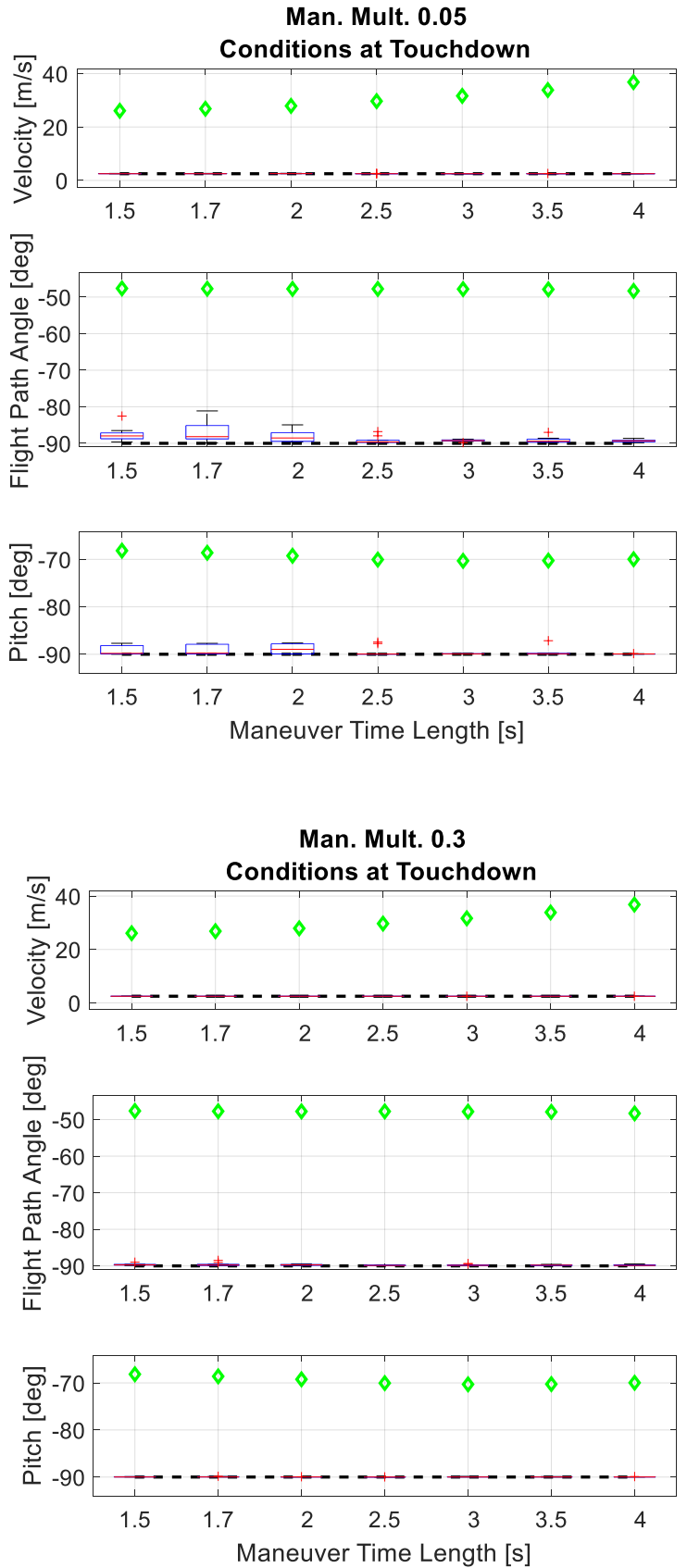

Figure 5. Maneuver variable effects on PDV's ability to meet the target conditions (planet relative velocity, flight path angle, and pitch angle) at discrete events. The above plots compare PDV operating nominally to one experiencing a loss of thrust in engine four and with no plant model update and to a PDV implementing a planet model update using the corresponding maneuver. a) Maneuver throttle multiplier of 0.05 . b) Maneuver throttle multiplier of 0.3. The box-and-whiskers plots divided by: Top Whisker (95\%-tile), Top of Box (75\%-tile), Red Line (50\%-tile), Bottom of Box (25\%-tile), and Bottom Whisker (5\%-tile).

conditions (right column in Figure 5a). Furthermore, the maneuver combination, that theoretically provides the least data content ( $1.5 \mathrm{~s}$ with 0.05 throttle multiplier), still provides enough information to enable the control system to compensate for the failed engine number four and land the PDV within a close proximity of the target conditions. Specifically, it is able to land within $0.1 \mathrm{~m} / \mathrm{s}$ of the velocity, $3^{\circ}$ of the flight path angle, $2^{\circ}$ and of the pitch angle targets. 
In looking at Figure 5b, the increase in throttle multiplier (0.3) increases the PDV's ability to reach the target conditions. This is again attributed to the increase data content, which decreases the model fit error, and improves the controller's ability to control the PDV. However, this increase in data content comes at the cost of utilizing more thrust and more resources. Lastly, there is a small increase in the pitch angle error at the vertical descent initiation as the maneuver time length is increased. This is due to the small deviations from the overall commanded inputs caused by the orthogonal multi-sine inputs. Even though these inputs are designed to minimize the overall deviation from the commanded, their effect becomes more noticeable with longer maneuvers. However, even for the $4 \mathrm{~s}$ maneuver, the effect on the pitch angle at vertical descent initiation are small at approximately $3.2^{\circ}$ off of the nominal $-90^{\circ}$. For reference, the InSight Mars Lander, scheduled to launch in May 2018, is the next robotic lander to Mars. The touchdown condition requirements for this lander under nominal operating conditions are: horizontal velocity less than $1.4 \mathrm{~m} / \mathrm{s}$, vertical velocity between $1.4-3.4 \mathrm{~m} / \mathrm{s}$, and pitch to be less than $5^{\circ}$ off vertical ${ }^{\ddagger}$. The Apollo Lunar Lander touchdown attitude requirement was to be $\leq 6^{\circ}$ of the local gravity vector ${ }^{15}$. Future human missions to Mars will likely have different requirements on their touchdown conditions. However, these two examples provide existing references to mission requirements imposed on PDVs landing a payload. Using them demonstrates that the failure mitigation strategy is able to land a PDV suffering from an engine failure to its touchdown conditions within reasonable bounds.

\section{B. IMU Error Study}

This study investigates the degradations in plant model estimation due to IMU noise, bias, and scale factor errors and the corresponding effects on the PDV's ability to reach its target conditions. It is assumed that all other sources of IMU errors are zero. The range of IMU errors investigated are taken from the LDSD Gimbaled LN-200 with Miniature Airborne Computer (GLN-MAC), the MSL Miniature Inertial Measurement Unit (MIMU), and the Honeywell HG9900 $0^{2,8,16}$. In this study, the data generated by the IMU is smoothed before entering the SLSFD routine.

Through the maneuver study discussed in Section V.A, a top performing version of the orthogonal multi-sine input function was found for each maneuver time length. Each of these are then applied to a 2000 case Monte Carlo simulation where only the IMU errors are dispersed. The results of the Monte Carlo simulation are used to show the correlations between the IMU errors and the PDV's ability to reach the target conditions. For brevity, only one engine failure scenario (full loss of thrust in engine three) along with two of the maneuvers ( $1.5 \mathrm{~s}$ long maneuver with 0.05 throttle multiplier and 4.0 long maneuver with 0.3 throttle multiplier) are shown.

Correlation coefficients provide a measure of the linear relationship between the investigated IMU errors and their effects on the plant model fit errors, which in turn affects the PDV's ability to reach the target touchdown conditions. Table 2 and Table 3 demonstrate the capability of the adaptive control allocation strategy implementing the maneuver with the least data content ( $1.5 \mathrm{~s}$ long maneuver with 0.05 throttle multiplier). Table 2 list the correlation coefficients, and Table 3 shows the impact on the model fit error and the errors in the PDV touchdown conditions. Table 4 and Table 5 provide the same series of results except the adaptive control allocation strategy is implementing the $4.0 \mathrm{~s}$ maneuver with a 0.3 throttle mutlipler, which has the most data content.

Table 2 shows that the IMU accelerometer and gyroscope noise have the highest correlations to the plant model fit error, thus impacting the PDV performance the most. However, Table 3 shows the overall impact to the model error is small. Thus the adaptive control allocation strategy is able to correctly identify the failed engine and generate an accurate planet model, which then allows the PDV to reach its target touchdown conditons.

Table 2. Correlation coefficients between the IMU sensor errors to the model error and touchdown conditions reached by the PDV.

\begin{tabular}{l|c|c|c|c|c|c} 
& \multicolumn{3}{|c|}{ Accelerometer } & \multicolumn{3}{c}{ Gyroscope } \\
\cline { 2 - 7 } & Noise & Bias & Scale Factor & Noise & Bias & Scale Factor \\
\hline Model Fit Error & 0.959 & -0.015 & -0.004 & 0.242 & 0.005 & -0.020 \\
\hline TD Velocity & 0.008 & -0.053 & 0.040 & 0.437 & 0.010 & -0.011 \\
\hline TD Pitch & -0.252 & 0.036 & 0.017 & 0.009 & -0.041 & 0.003 \\
\hline TD Flight Path Angle & 0.836 & 0.016 & 0.013 & 0.455 & 0.001 & -0.017
\end{tabular}

\footnotetext{
* Information provided through communication with Robert Maddock, NASA Langley EDL Lead for NASA InSight Mars Lander.
} 
Table 3. IMU sensor errors induce the below range of errors in the parameter identification plant model update and the PDV's ability to reach its target touchdown conditions of $\mathbf{2 . 5} \mathbf{~ m} / \mathrm{s}$ and $\mathbf{- 9 0 ^ { \circ }}$ pitch and flight path angles.

\begin{tabular}{l|c|c|c}
\multirow{2}{*}{} & \multicolumn{3}{|c}{ Errors } \\
\cline { 2 - 4 } & Min & Mean & Max \\
\hline Model Fit Error & 0.08 & 0.12 & 0.17 \\
\hline TD Velocity [m/s] & 0.00 & 0.00 & 0.00 \\
\hline TD Pitch [deg] & 0.01 & 0.04 & 0.10 \\
\hline TD Flight Path Angle [deg] & 0.86 & 1.15 & 1.85
\end{tabular}

Table 4. Correlation coefficients between the IMU sensor errors to the model error and touchdown conditions reached by the PDV.

\begin{tabular}{l|c|c|c|c|c|c} 
& \multicolumn{3}{|c|}{ Accelerometer } & \multicolumn{3}{c}{ Gyroscope } \\
\cline { 2 - 7 } & Noise & Bias & Scale Factor & Noise & Bias & Scale Factor \\
\hline Model Fit Error & -0.106 & 0.360 & -0.484 & 0.741 & 0.001 & 0.222 \\
\hline TD Velocity & 0.031 & -0.009 & 0.056 & -0.025 & -0.006 & 0.024 \\
\hline TD Pitch & -0.304 & 0.834 & -0.080 & 0.039 & 0.006 & 0.042 \\
\hline TD Flight Path Angle & 0.506 & -0.791 & 0.025 & 0.363 & 0.002 & -0.036
\end{tabular}

Table 5. IMU sensor errors induce the below range of errors in the parameter identification plant model update and the PDV's ability to reach its target touchdown conditions of $2.5 \mathrm{~m} / \mathrm{s}$ and $\mathbf{- 9 0 ^ { \circ }}$ pitch and flight path angles.

\begin{tabular}{l|c|c|c}
\multirow{2}{*}{} & \multicolumn{3}{|c}{ Errors } \\
\cline { 2 - 4 } & Min & Mean & Max \\
\hline Model Fit Error & 0.02 & 0.02 & 0.03 \\
\hline TD Velocity [m/s] & 0.00 & 0.00 & 0.00 \\
\hline TD Pitch [deg] & 0.00 & 0.01 & 0.02 \\
\hline TD Flight Path Angle [deg] & 0.07 & 0.14 & 0.20
\end{tabular}

Table 4 provides results for the adaptive control allocation strategy implementing a maneuver that provides the most data content ( $4.0 \mathrm{~s}$ long maneuver with 0.3 throttle multiplier). Although Table 4 shows the IMU accelerometer and gyroscope bias and scale factors play a larger role in the plant model fit error, Table 5 shows the net impact is significantly reduced when compared to Table 3 .

\section{Engine Failure Scenarios}

Up to this point results have been focused on a PDV experiencing a lss of thrust in one engine. Figure 6 shows the broader application of the adaptive control allocation strategy to single engine failure scenarios: loss of thrust, thrust stuck full on, and 50\% loss of thrust. In each of these failure scenarios, the simulated PDV with no adaptive control is shown in the left column; the PDV with adaptive control is in the right. The loss of thrust scenario (Figure 6a, left column) causes the PDV with no adaptive control to impact the ground at $15.5 \mathrm{~m} / \mathrm{s}$ and at a flight path angle of $-59.9^{\circ}$. In the stuck full on scenario (Figure 6b, left column), the simulated PDV tumbles until it impacts the ground at nearly $25 \mathrm{~m} / \mathrm{s}$. In the $50 \%$ loss of thrust scenario (Figure 6c, left column), the PDV is able to meet the $2.5 \mathrm{~m} / \mathrm{s}$ touchdown velocity condition, but its flight path angle is $-79.1^{\circ}$. In all failure scenarios where the PDV implements the adaptive control allocation strategy, the PDV is able to meet the target conditions without tumbling or loss of control. 
PDV No Adaptive Control

Angles vs. Time

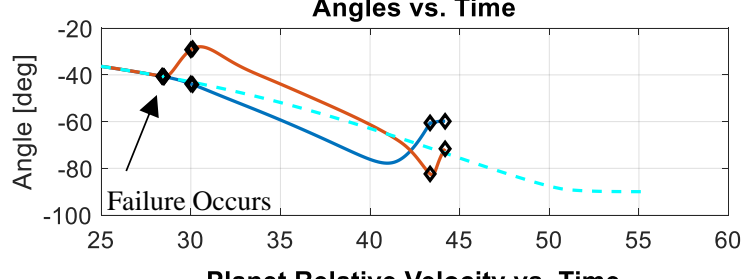

Planet Relative Velocity vs. Time
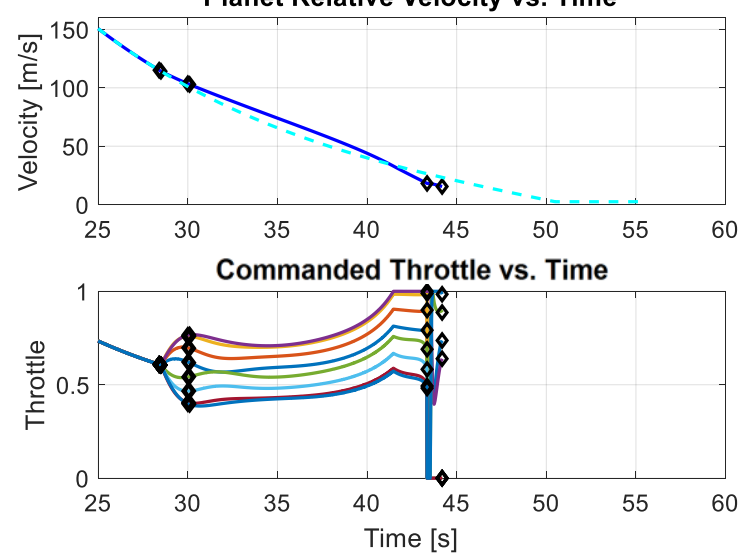

PDV With Adaptive Control

Angles vs. Time
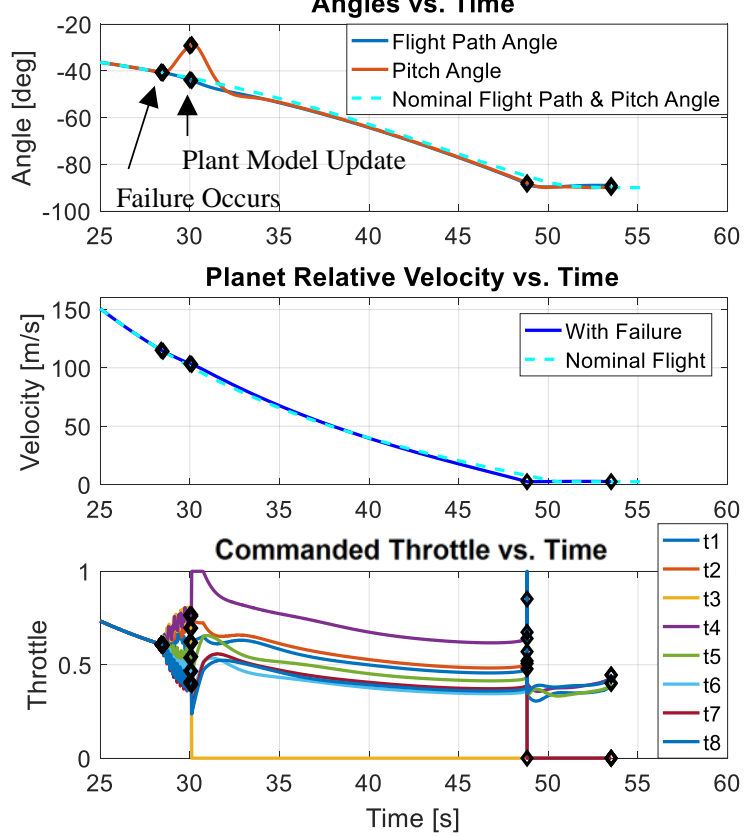

a) Loss of thrust in engine three.
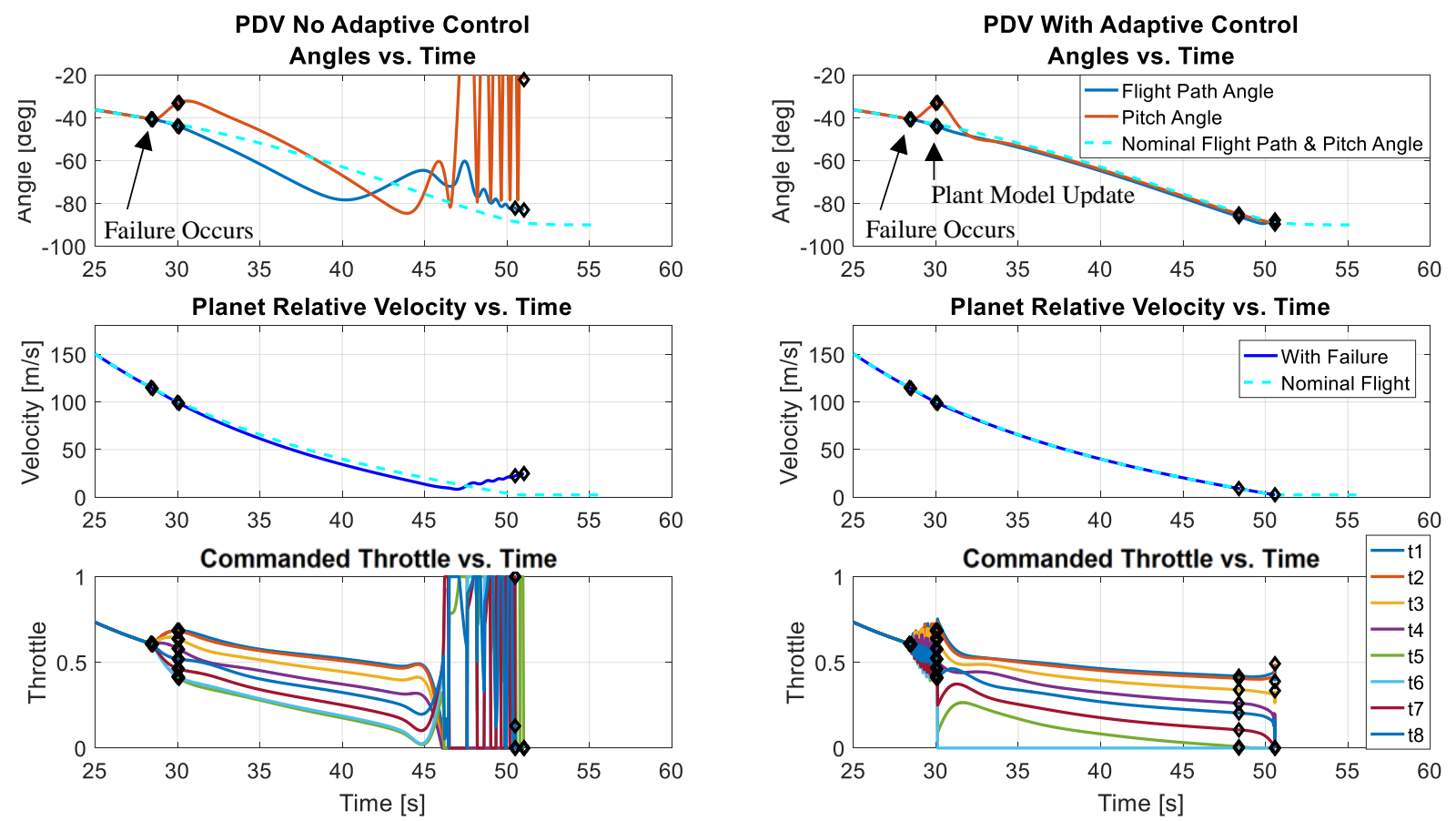

b) Engine six is stuck at full thrust. 

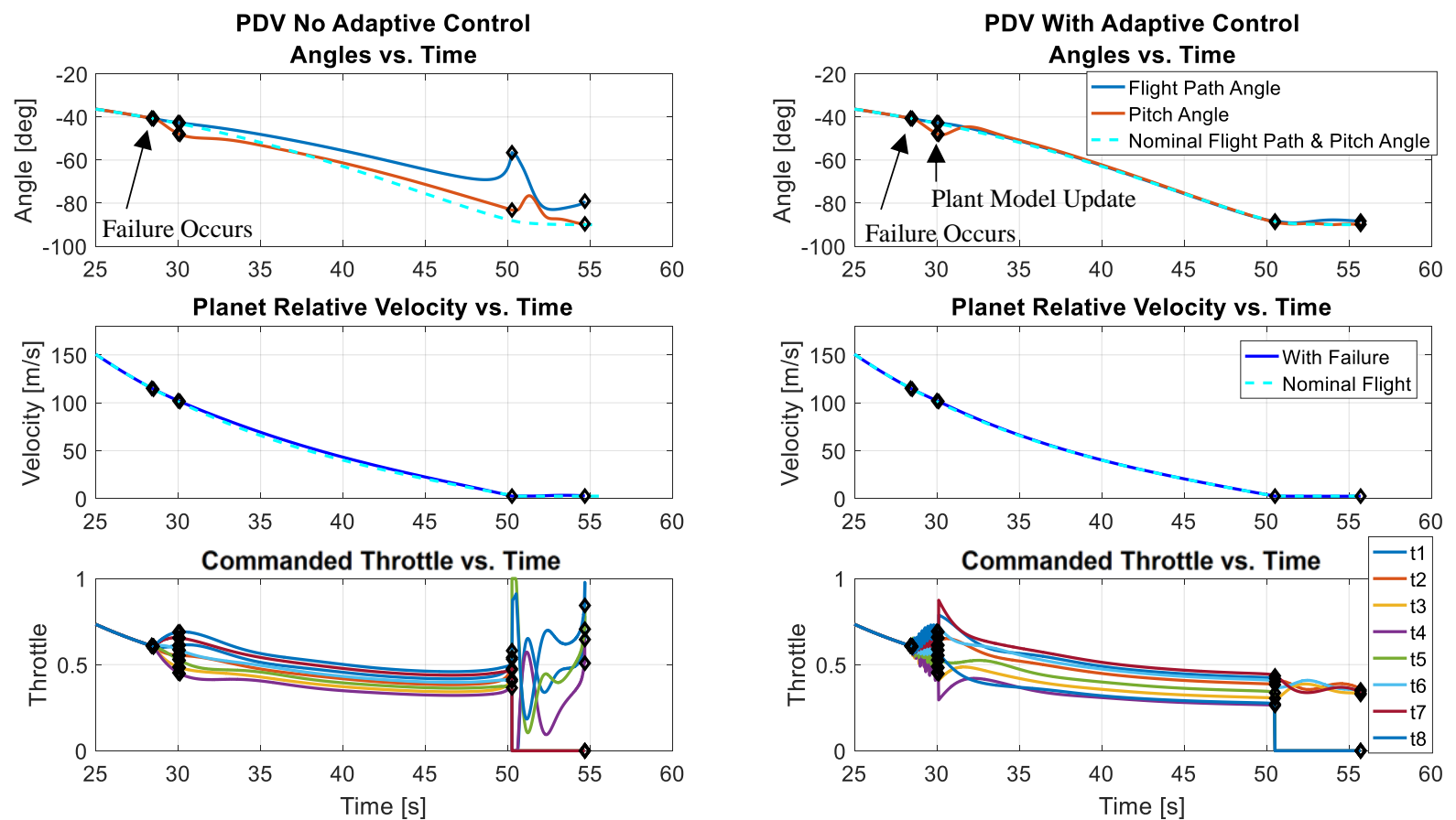

c) Engine eight is only able to produce $50 \%$ of the commanded thrust.

Figure 6. This figure demonstrates the flight of a simulated 46.4mt PDV equipped with eight fixed $100 \mathrm{kN}$ engines. In the left column, the PDV experiences a single engine failure and has no adapting capabilities; in the right column the same PDV experiences the same failure, but is using the adaptive control allocation strategy discussed in this paper. Three failure scenarios shown are: a) full loss of thrust in engine three, b) engine six is stuck full on, and c) engine eight is only able to produce $50 \%$ of the commanded thrust. The dashed cyan lines represent the nominal flight of the PDV (no engine failure). The throttle plots in parts a), b), and c) show the throttle commands to each of the eight engines. Note that the throttle plots in the left columns of parts a), b), and c) show continued commands to the failed engines even though they are not responsive. However, the throttle plots in the right columns, where the adaptive control allocation strategy is implemented, discontinue the commands to the failed engines and the other seven engines are adjusted to make up for the loss of thrust.

\section{Conclusion}

The goal of this research is to enable future PDVs to adapt in real-time to failures and degradations in their performance. The real-time adaptive control allocation is performed using SLSFD on-board combined with a maneuver to estimate the PDV plant model. The ability to generate a new plant model on-board enables the PDV to identify underperforming and failed engines. This information is fed to the guidance and control systems where it adapts the engine commands to mitigate the failure.

The work herein explores the design space of a maneuver to assist on-board identification of engine failures. Although the plant model fit error is lower for longer and larger amplitude maneuvers, the $1.5 \mathrm{~s}$ and $5 \%$ throttle amplitude maneuver is found to provide sufficient data for the SLSFD to generate a plant model, while impacting the PDV flight the least. Additionally, the combination of the chosen maneuver with SLSFD were found to be robust to IMU errors.

This approach provides a predominantly software approach to failure mitigation that does not rely on duplicate hardware, thus saving mass and system complexity. Additionally, this work focuses on the use of IMU measured data to identify the specific engine and type failure it is experiencing. This approach is effective in adapting the on-board control of the PDV to mitigate an engine failure. Future EDL missions can implement this adaptive control allocation strategy to enable their powered descent vehicle to land in the event of an engine failure without the loss of crew or assets. Additionally, this work can be readily applied to non-EDL flight systems, such as commercial quadcopters.

Several topics are planned for future work. The first is to test the performance of the failure mitigation strategy using a navigational filter versus the smoothing routine, which was applied here. The second, will be to implement a detection strategy that will be used to initiate the failure mitigation strategy discussed here. Third, to investigate the 
effects engine dynamics have on the accuracy of the parameter estimates used to update the plant model. Lastly, to investigate the effects to the SLSFD method due to aerodynamic interactions induced by the engine plume on the freestream flow.

\section{Acknowledgments}

The authors acknowledge the support of Dr. Juan Cruz, Dr. Eric Queen, Dr. Eugene Morelli, and Dr. Jared Grauer from NASA Langley Research Center; and the support of Richard Powell and James Hoffman from Analytical Mechanics Associates, Inc.

\section{References}

\footnotetext{
${ }^{1}$ Drake, B. G., "Human Exploration of Mars Design Reference Architecture 5.0,” NASA-SP-2009-566, July 2009.

${ }^{2}$ Weiss, J. M., and Guernsey, C. S., "Design and Development of the MSL Descent Stage Propulsion System," AAS/AIAA Space Flight Mechanics Meeting, Kauai, 2013, AAS 13-458.

${ }^{3}$ Adler, M., Wright, M., Campbell, C., Clark, I., Engelund, W., and Rivellini, T., "Entry, Descent, and Landing Roadmap Technology Area 09," National Aeronautics and Space Administration, Washington DC, April 2012.

${ }^{4}$ Striepe, S. A., Powell, R. W., Desai, P. N., Queen, E. M., Way, D. W., Prince, J. L., Cianciolo, A. M., Davis, J. L., Litton, D. K., Maddock, R. M., Shidner, J. D., Winski, R. G., O’Keefe, S. A., Bowes, A. G., Aguirre, J. T., Garrison, C. A., Hoffman, J. A., Olds, A. D., Dutta, S., Zumwalt, C. H., White, J. P., Brauer, G. L., Marsh, S. M., Lugo, R. A., Green, J. S., "Program To Optimize Simulated Trajectories II (POST2): Utilization Manual,” Vol. 2, Ver. 4.0.0.r1173, July 2017.

${ }^{5}$ Polsgrove, T., Chapman, J., Sutherlin, S., Taylor, B., Fabisinski, L., Collins, T., Dwyer-Cianciolo, A., Samareh, J., Robertson, E., Studak, B., Vitalpur, S., Lee, A., Rakow, G., "Human Mars Lander Design for NASA's Evolvable Mars Campaign," IEEE Aerospace Conference, March 2016.

${ }^{6}$ Dwyer-Cianciolo, A., Polsgrove, T., "Human Mars Entry, Descent, and Landing Architecture Study Overview," AIAA SPACE Conferences and Exposition, September 2016, AIAA 2016-5494.

7 Justh, H. L., “Mars Global Reference Atmospheric Model 2010 Version: Users Guide,” NASA/TM-2014-217499, 2014.

${ }^{8}$ Karlgaard, C. D., O’Farrell, C., Ginn, J. M., Van Norman, J. W., "Supersonic Flight Dynamics Test 2: Trajectory Atmosphere, and Aerodynamics Reconstruction," AAS/AIAA Spaceflight Mechanics Meeting, Napa, 2016. AAS 16-217

${ }^{9}$ V. Klein, E. A. Morelli, Aircraft System Identification: Theory and Practice, AIAA Education Series, AIAA, Reston, VA, 2006.

${ }^{10}$ Brandon, J. M., Derry, S. D., Heim, E. H., Hueschen, R. M., Bacon, B. J., “Ares-I-X Stability and Control Flight Test: Analysis and Plans," AIAA Space 2008 Conference \& Exposition, San Diego, 2008, AIAA 2008-7807.

${ }^{11}$ Grauer, J., “Aircraft Fault Detection using Real-Time Frequency Response Estimation,” in AIAA Guidance, Navigation, and Control Conference, San Diego, 2016, AIAA 2016-0372.

${ }^{12}$ Song, Y., Campa, G., Napolitano, M., Seanor, B., and Perhinschi, M. G., "Online Parameter Estimation Techniques Comparison Within a Fault Tolerant Flight Control System," Journal of Guidance, Control, and Dynamics, vol. 25, no. 3, pp. 528-537, 2002.

${ }^{13}$ SIDPAC, System IDentification Programs for AirCraft, Software Package, Ver. 3.0, E. A. Morelli, Hampton, VA, 2014.

${ }^{14}$ Morelli, E. A., "Multipler Input Design for Real-Time Parameter Estimation in the Frequency Domain," $13^{\text {th }}$ IFAC Conference on System Identification, Vol. 36, No. 16, Rotterdam, 2003, REG-360.

${ }^{15}$ Rogers, W. R., “Apollo Experience Report - Lunar Module Landing Gear Subsystem,” NASA TN D-6850, 1972.

${ }^{16}$ Honeywell Aerospace, HG9900 Navigation-Grade Inertial Measurement Unit (IMU), N61-0491-000-001, September 2009.
} 